\title{
Influência de fragmentos florestais sobre a nidificação de vespas sociais (Hymenoptera, Vespidae) em cafeeiro
}

\author{
Lucas Rocha Milani ${ }^{1}$, Gabriel de Castro Jacques ${ }^{2 *}$, Mateus Aparecido Clemente ${ }^{3}$, Evando Luiz \\ Coelho $^{4} \&$ Marcos Magalhães de Souza ${ }^{4}$
}

\author{
${ }^{1}$ Universidade Federal de Juiz de Fora, Juiz de Fora, MG, Brasil. \\ ${ }^{2}$ Instituto Federal Educação, Ciência e Tecnologia de Minas Gerais, IFMG Campus Bambuí, Bambuí, MG, Brasil. \\ ${ }^{3}$ Universidade Estadual Paulista, Unesp Campus Rio Claro, Rio Claro, SP, Brasil. \\ ${ }^{4}$ Instituto Federal de Educação, Ciência e Tecnologia do Sul de Minas Gerais, Campus Inconfidentes, Inconfidentes, MG, Brasil.
}

*E-mail para correspondência: gabriel.jacques@ifmg.edu.br

\begin{abstract}
RESUMO
O desmatamento imposto por culturas agrícolas afeta negativamente as interações ecológicas protagonizadas por determinadas espécies, como as vespas sociais. Uma hipótese é que o tamanho dos fragmentos florestais e sua proximidade destas culturas podem influenciar na nidificação das vespas sociais na área de plantio. Objetivou-se, portanto, testar esta hipótese. O trabalho ocorreu em Inconfidentes e Ouro Fino, Minas Gerais, Brasil. O método utilizado foi a busca ativa, percorrendo os fragmentos de mata e áreas associadas por um período de 32 dias, distribuídos entre janeiro de 2014 e janeiro de 2016. Foram amostradas duas áreas de plantio de café (A1 com 25 hectares e A2 com cinco) associadas a fragmentos de tamanhos diferentes. Foram registradas 55 colônias de 20 espécies. Na cultura associada a um sistema ecológico mais diversificado (A1), as vespas sociais estabeleceram suas colônias na planta do café. Na cultura associada a fragmentos menores e sistema ecológico mais simplificado (A2), elas apresentaram dificuldade de nidificação no cafezal. Entretanto, mesmo o fragmento florestal menor associado à A2 mostrou-se relevante para a manutenção das vespas sociais, evidenciando a importância da manutenção de fragmentos florestais próximo às culturas.
\end{abstract}

Palavras-chave: Coffea arabica, Controle biológico, Nidificação, Polistinae

\section{ABSTRACT}

Influence of forest fragments on the nesting of social wasps (Hymenoptera, Vespidae) in coffee plant. Deforestation imposed by agricultural crops negatively affects ecological interactions carried out by certain species, such as social wasps. One hypothesis is that the size of the forest fragments and their proximity to these crops may influence the nesting of social wasps in the crop area. Therefore, the objective was to test this hypothesis. The study was made in Inconfidentes and Ouro Fino, Minas Gerais, Brazil. The method used was an active search, covering the forest fragments and associated areas for 32 days, distributed between January 2014 and January 2016. It was sampled two coffee plantation areas (A1 with 25 hectares and A2 with five hectares), both associated with forest fragments with different sizes. We recorded 55 colonies with 20 species. In the crop associated with a more diversified ecological system (A1), the social wasps were able to establish their colonies in the coffee plant. In the crop associated with smaller fragments and more simplified ecological system (A2), the social wasps presented difficulty on nesting on the coffee plant. Nevertheless, even the forest fragment associated with A2 being smaller, it was relevant for the maintenance of social wasps, which evidences the importance of the maintenance of forest fragments near the crops.

Keywords: Biological control, Coffea arabica, Nesting, Polistinae 


\section{INTRODUÇÃO}

A Floresta Estacional Semidecidual Montana também chamada de Floresta Atlântica do interior ocorre acima de 500 metros de altitude em diversos estados do Brasil, e em Minas Gerais, perfaz em torno de $9 \%$ da cobertura vegetal, constituindo a vegetação predominante no sul do estado (Machado et al., 2004). Entretanto, este ecossistema vem se fragmentando com o avanço das culturas, em especial a do café Coffea arabica L. (Rubiaceae), fortemente difundida na região sul de Minas Gerais, que é a maior produtora do Brasil (Zanella et al., 2012; EMBRAPA, 2019).

A fragmentação das florestas afeta negativamente diferentes serviços ambientais, como polinização, visitação floral e também o controle biológico (Altieri, 1994) por promover o isolamento das populações, tendo como exemplo os predadores naturais, alterando assim a habilidade destes em se dispersar, o que resulta em uma redução no tamanho das populações regionais (Jonsen \& Fahrig, 1997), podendo favorecer positivamente diferentes espécies de pragas na cultura do café e ocasionar perdas ao produtor (Fernandes et al., 2009). Sendo assim, a manutenção de áreas naturais associadas à área de cultivo pode auxiliar no controle natural de pragas em diversas culturas, incluindo o cafezal, pois fornece abrigo aos inimigos naturais (Altieri, 1994; Altieri \& Silva, 2003; Hoshino et al., 2018).

A cultura de café é atacada por diversos insetos que, se não forem devidamente controlados, ocasionam grandes prejuízos, como por exemplo, perfurações das folhas, sucção da seiva, introdução de toxinas, entre outras ações que ocasionam a queda da folha e redução da produtividade (Bartra, 1974; Nais \& Busoli, 2012). Dentre as espécies que causam prejuízos aos cafeeiros destaca-se o bicho-mineiro Leucoptera coffeella (Guérin-Mèneville, 1842) (Lepidoptera: Lyonetiidae) (Reis et al., 2002; Righi et al., 2013), sendo que o controle desta praga pode ser realizado pelo controle biológico exercido por inimigos naturais, como as vespas sociais (Tuelher et al., 2003; Barros et al., 2015; Hoshino, et al., 2018).

Estas vespas pertencem à ordem Hymenoptera, família Vespidae, subfamília Polistinae, e são importantes no controle biológico de sistemas naturais e agrossistemas, pois agem como predadores de insetos das ordens Diptera, Hemiptera, Hymenoptera e, principalmente, larvas de Lepidoptera, que compreendem cerca de 90 a 95\% das presas capturadas (Marques, 1996; Prezoto \& Machado, 1999; Andrade \& Prezoto, 2001; Scalon et al., 2011). Algumas espécies de vespas sociais como Brachygastra lecheguana Latreille, 1824, Polybia occidentalis (Olivier, 1971) e Polybia paulista (H. von Ihering, 1896) atuam na predação de L. coffeella, principal praga do café (Perioto et al., 2011).

Diferentes espécies de vespas sociais já foram registradas em cafezais (Freitas et al., 2015), contudo não se sabe os fatores que levam estes insetos a nidificarem nesta cultura e em seu entorno, bem como a influência do tamanho dos fragmentos florestais sobre estas nidificações. Porém, compreende-se que 
áreas de plantio de café associadas a fragmentos florestais apresentam maior abundância de vespas sociais quando comparadas a áreas sem a presença de fragmentos (Freitas et al., 2015).

Desta maneira, testamos a hipótese de que fragmentos florestais associados a culturas de café podem influenciar na presença de colônias de vespas sociais na área de plantio, e que quanto maior a extensão destes fragmentos, melhor a manutenção da comunidade destes insetos.

\section{MATERIAL E MÉTODOS}

O trabalho foi realizado nos municípios de Inconfidentes e Ouro Fino, sul do estado de Minas Gerais, Brasil. As coletas ocorreram em duas áreas de cultivo de café (A1 e A2), com manejo similar quanto à aplicação de produtos fitossanitários e colheita, estando ambas associadas a fragmentos florestais que são paralelos ao cultivo. Foram 32 dias amostrais (16 para cada cultivo) distribuídos entre o período de Janeiro de 2014 e Janeiro de 2016.

A área A1 (2217’39,25”S 4620’35,20”O) (Figura 1A) está associada a 25 hectares de fragmentos florestais, presença de cultura de banana (Musa parasidiaca L.) e vegetação ruderal nas ruas de café. A área A2 (2218'17,80"S 4617'32,02"O) (Figura 1B) está associada a fragmentos que somam uma extensão de 5 hectares, porém não existem culturas associadas e praticamente não há plantas ruderais nas ruas de café, estabelecendo um sistema agrícola com vegetação menos diversificada. Estas áreas foram subdivididas da seguinte maneira: a área A1 formada pelas subáreas cafezal 1 (C1) e fragmento 1 (F1), e área $\mathrm{A} 2$ pelas subáreas cafezal 2 (C2) e fragmento 2 (F2).

O método utilizado para registro de colônias foi a busca ativa de maneira aleatória, com o auxílio de redes entomológicas e câmaras mortíferas, cobrindo completamente as duas áreas ao final das coletas, deslocando pelas ruas de café, borda e interior dos fragmentos por trilhas pré-existentes. Espécimes de cada colônia foram coletadas com rede entomológica e identificadas com auxílio de chaves entomológicas (Richards, 1978; Cooper, 1997; Pickett \& Wenzel, 2007) e comparadas com a coleção do Instituto Federal de Educação, Ciência e Tecnologia do Sul de Minas Gerais, Campus Inconfidentes.

A diversidade foi calculada com o índice de Shannon-Wiener $\left(\mathrm{H}^{\prime}\right)$, a dominância com o índice de Berger-Parker $\left(D_{\mathrm{pb}}\right)$, e a similaridade com teste de Kruskal-Wallis, com grau de confiabilidade de $95 \%$ através do programa Past (Hammer et al., 2005). 


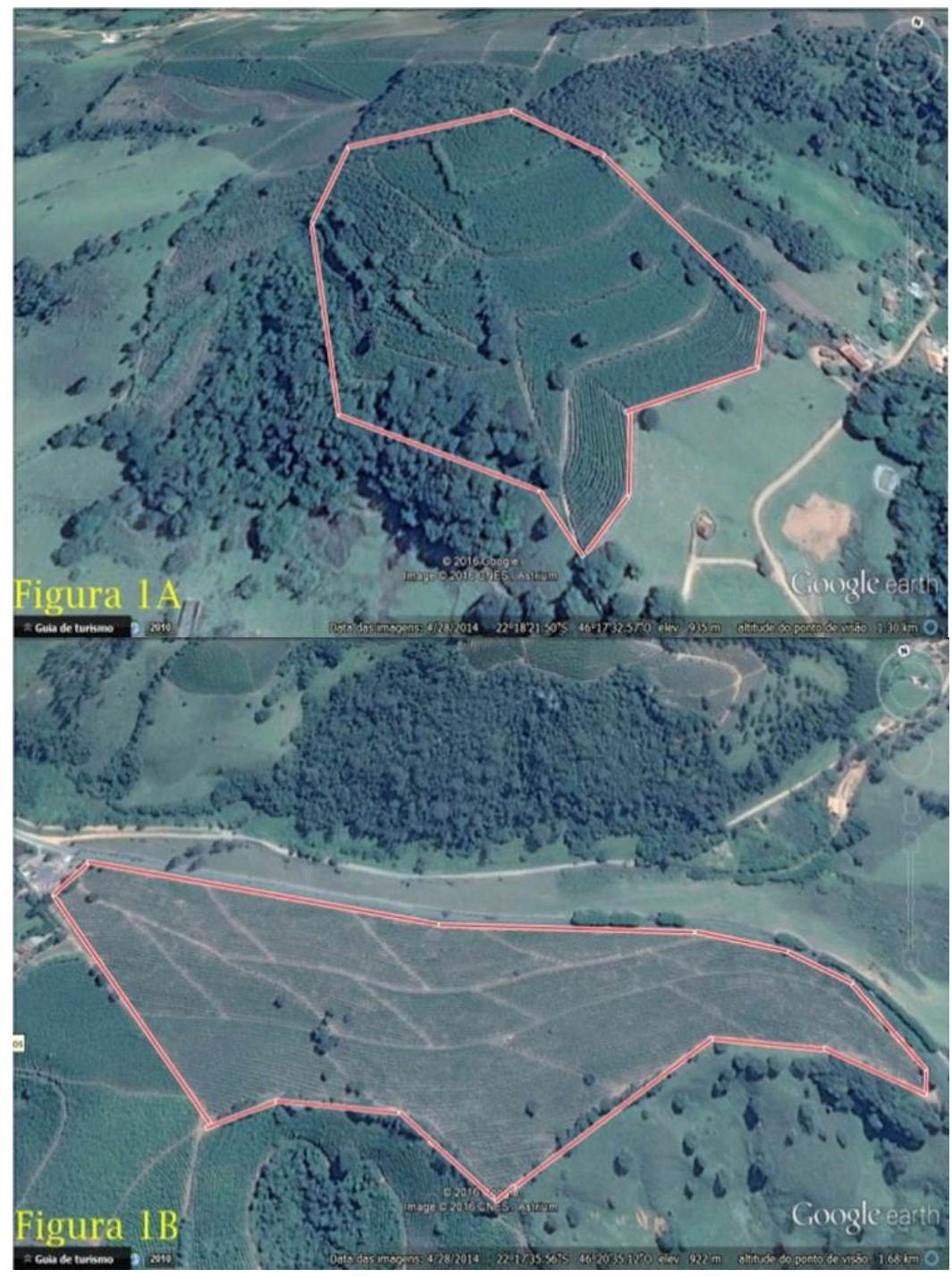

Figura 1. Áreas amostradas para registro de colônias de vespas sociais em cultivo de café nos municípios de (A) Inconfidentes; (B) Ouro Fino, sul de Minas Gerais, Brasil. Fonte. Google Earth.

\section{RESULTADOS E DISCUSSÃO}

Foram registradas 55 colônias de 20 espécies e sete gêneros de vespas sociais, sendo Polybia paulista (H. von Ihering, 1896), a espécie com maior número de colônias ( $n=6)$ (Tabela 1). Essa espécie é frequente em ambientes naturais (Souza \& Prezoto, 2006; Albuquerque et al., 2015), agrícolas (Perioto et al., 2011; Jacques et al., 2015) e sinantrópicos (Canevazzi \& Noll, 2011; Vinhote et al., 2011; Jacques et al., 2012; Oliveira et al., 2017), e somado ao fato da mesma predar Leucoptera coffeella (Guérin-Mèneville, 1842) (Lepidoptera, Lyonetiidae), torna a presença dessa espécie valiosa para o controle biológico no café (Fragoso et al., 2001). 
Tabela 1. Riqueza, diversidade e dominância de espécies de vespas sociais coletadas em duas áreas de cultivo de café nos municípios de Inconfidentes (A1) e Ouro Fino (A2). As duas áreas foram divididas em duas subáreas cada: A1 em Cafezal 01 (C1) e Fragmento 01 (F1); A2 em Cafezal 02 (C2) e Fragmento 02 (F2).

\begin{tabular}{|c|c|c|c|c|}
\hline \multirow[b]{3}{*}{ Espécies } & \multicolumn{4}{|c|}{ Número de colônias registradas } \\
\hline & \multicolumn{2}{|c|}{ A1 } & \multicolumn{2}{|c|}{$\mathrm{A} 2$} \\
\hline & C1 & F1 & $\mathrm{C} 2$ & $\mathrm{~F} 2$ \\
\hline Agelaia multipicta (Haliday, 1836) & - & - & - & 1 \\
\hline Agelaia pallipes Olivier, 1792 & 2 & - & - & - \\
\hline Apoica gelida (Van Der Vecht, 1973) & - & 2 & - & 1 \\
\hline Mischocyttarus socialis (Saussure, 1854) & - & - & - & 4 \\
\hline $\begin{array}{l}\text { Mischocyttarus cassununga (R. Von. Ihering, } \\
\text { 1903) }\end{array}$ & 1 & - & - & 4 \\
\hline Mischocyttarus cerberus (Richards, 1940) & - & - & - & 1 \\
\hline Mischocyttarus drewseni (Saussure, 1857) & 2 & - & - & - \\
\hline Parachartergus fraternus (Gribodo, 1892) & - & - & 1 & - \\
\hline Polistes actaeon (Haliday, 1836) & 2 & - & - & - \\
\hline Polistes ferreri (Saussure, 1853) & - & - & - & 1 \\
\hline Polistes simillimus (Zikán, 1951) & 2 & - & - & 2 \\
\hline Polistes versicolor (Olivier, 1971) & 5 & - & - & - \\
\hline Polybia chrysothorax (Lichtenstein, 1796) & 1 & - & - & 2 \\
\hline Polybia fastidiosuscula (Saussure, 1854) & 1 & 2 & - & - \\
\hline Polybia occidentalis occidentalis (Olivier, 1971) & 1 & - & 2 & 2 \\
\hline Polybia paulista (H. von Ihering, 1896) & - & 1 & 4 & 1 \\
\hline Polybia platycephala slyventris (Richards, 1978) & - & 1 & - & - \\
\hline Polybia sericea (Olivier, 1791) & - & 1 & - & - \\
\hline Protopolybia sedula (Saussure, 1854) & 4 & - & - & - \\
\hline Synoeca cyanea (Fabricius, 1775) & - & - & - & 1 \\
\hline Número de colônias & 21 & 7 & 7 & 20 \\
\hline Riqueza de espécies (S`) & 10 & 5 & 3 & 11 \\
\hline Índice de Shannon-Wiener ( $\left.\mathrm{H}^{\prime}\right)$ & $2,13^{a}$ & $1,55^{b}$ & $0,95^{c}$ & $2,12^{\mathrm{a}}$ \\
\hline Índice de Berger-Parker (Dpb) & 0,238 & 0,285 & 0,571 & 0,222 \\
\hline
\end{tabular}

${ }^{a-c}$ Linhas com letras diferentes diferem entre si pelo teste de Tukey a $5 \%$ de significância.

Houve o registro de colônias das espécies Agelaia pallipes (Olivier, 1972), Polybia occidentalis occidentalis (Olivier, 1791), Polybia sericea (Olivier, 1971) e Synoeca cyanea (Fabricius, 1775) (Tabela 1), que já foram relatadas como eficientes predadores das pragas do café (Parra et al., 1977; Reis \& Souza, 2002; Perioto et al., 2011). Além disso, foram encontradas colônias de quatros espécies do gênero Polistes, que se destaca como um importante agente de controle biológico, pois são excelentes predadores de pragas agrícolas, incluindo as do café (Gravena, 1983; Prezoto et al., 2006; Elisei et al., 2010).

Não houve diferença significativa em relação ao índice de diversidade $(p=0,206)$ e similaridade $(p=0,189)$ entre as áreas A1 e A2. Porém se observou diferença significativa entre as subáreas. A subárea C2, que é uma cultura de café associada a fragmentos menores e sistema ecológico mais simplificado, 
obteve menor índice de diversidade, com uma baixa riqueza de espécies ( $n=3$ ) e o número de colônias encontradas $(n=7)$. Esse ambiente mais homogêneo pode ter dificultado a nidificação das vespas sociais no próprio cafeeiro. Sabe-se que, em geral, a vegetação é o principal substrato para a fundação de colônias de vespas sociais sendo que os ambientes heterogêneos podem aumentar a coexistência de um número maior de espécies (Souza et al., 2012). Diversos estudos mostram que ambientes estruturalmente complexos possuem mais nichos para o estabelecimento e sobrevivência de um número maior de espécies (Lawton, 1983; Santos et al., 2000; Santos et al., 2007; Souza et al., 2010; Bueno et al., 2019).

A estrutura da vegetação influencia diretamente nas comunidades de vespas sociais, pois além de fornecer apoio e materiais para a construção dos ninhos, recursos glicídicos, recursos para a área de caça a vegetação pode influenciar a quantidade de sombreamento, temperatura e umidade do ar, afetando assim as comunidades de vespas sociais. Algumas espécies apenas nidificam sob certas condições estruturais, selecionando as formas abertas ou fechadas e os tipos de estrutura da vegetação (como forma e arranjo das folhas) (Naumann, 1975; Dejean et al., 1998; Santos et al., 1998; Francisco et al., 2018). Além disso, C2 foi a subárea que teve o maior índice de dominância ( $\mathrm{Dpb}=0,571)$ ( $P$. paulista), o que leva uma diminuição no índice de diversidade.

Já a subárea (C1) teve, junto com a subárea (F2), o maior índice de diversidade. A subárea de cafezal (C1) forma um ambiente mais heterogêneo, com a presença da cultura de banana e vegetações ruderais, além da proximidade de um fragmento de Floresta Atlântica com 25 hectares. Assim, as vespas sociais conseguiram estabelecer suas colônias na planta do café, afetando positivamente o número de espécies $(n=10)$ e colônias encontradas $(n=21)$. Esse ambiente heterogêneo fornece uma maior disponibilidade de micro habitats, proteção contra predadores, substrato para nidificação, disponibilidade e diversidade de recursos alimentares (Santos et al., 2007; Souza et al., 2012).

Em relação aos fragmentos, a subárea F2 mesmo sendo menor, apresentou um maior índice de diversidade em relação a F1 (Tabela 1). A área F2 pode ter atuado como um refúgio para a nidificação das vespas sociais locais, tendo em vista a ausência de heterogeneidade da área C2. Sabe-se ainda que as vespas sociais realizam diversos deslocamentos entre sítios de forrageio e as suas colônias (RaveretRichter, 2000) e, frequentemente, retornam onde o recurso é abundante (Nakasuji et al., 1976), situação que ocorre em cafezais, onde lagartas são encontradas. Desta forma, torna-se mais vantajoso para esses insetos nidificar próximo destes locais, resultando em menores gastos energéticos no forrageio. $\mathrm{A}$ área C1 ofereceu condições para que estes insetos se estabelecessem, provavelmente devido à presença espécies ruderais e cultura de bananas, tornando este sistema mais diversificado (Tomazella et al., 2018). 
Este fato, pode ter contribuído para uma menor dependência destes insetos sociais aos fragmentos associados, mesmo estes sendo relativamente maiores quando comparado a outra área de estudo (C2 e F2). A menor riqueza registrada $(\mathrm{N}=5)$ no fragmento com 25 hectares pode estar relacionada ao seu tamanho, o que gera mais prováveis locais para nidificação e dificulta os registros, quando comparado à área 2, com 5 hectares.

Diferentes estudos reportam que áreas de cultura associadas a outras culturas, fragmentos naturais próximos e plantas ruderais favorecem positivamente diferentes populações de inimigos naturais (Fragoso et al., 2001; Altieri \& Silva, 2003; Amaral et al., 2010; Tomazella et al., 2018) o que se alinha aos dados obtidos no presente estudo.

\section{CONCLUSÃO}

A manutenção de fragmentos florestais e de ecossistemas agrícolas diversificados associados à cultura de café influenciam positivamente na riqueza e nidificação das vespas sociais nas áreas associadas.

A vegetação é o principal substrato para a fundação de colônias de vespas sociais e, quando associada à heterogeneidade ambiental, permite a coexistência de um número maior de espécies. Dessa forma, a manutenção de fragmentos florestais e de ecossistemas agrícolas diversificados associados à cultura de café influenciará positivamente na riqueza e nidificação das vespas sociais, um importante inimigo natural de pragas da cafeicultura.

\section{AGRADECIMENTOS}

Ao Instituto Federal de Educação, Ciência e Tecnologia do Sul de Minas Gerais, Campus Inconfidentes por todo o suporte, aos cafeicultores por terem cedido às áreas de cultura e também a todos que ajudaram com as coletas.

\section{REFERÊNCIAS BIBLIOGRÁFICAS}

Albuquerque, C.H.B.; Souza, M.M. \& Clemente, M.A. 2015. Comunidade de vespas sociais (Hymenoptera, Vespidae) em diferentes gradientes altitudinais no sul do estado de Minas Gerais, Brasil. Biotemas 28(4): 131-138. 
Altieri, M.A. 1994. Biodiversity and pest management in agroecosystems. New York, Food Products Press. 185p.

Altieri, M.A. \& Silva, E.N.N. 2003. O papel da biodiversidade no manejo de pragas. São Paulo, Holos Editora. $215 p$.

Amaral, D.S.; Venzon, M.; Pallini, A.; Lima, P.C. \& Souza, O. 2010. A Diversificação da Vegetação Reduz o Ataque do Bicho-Mineiro-do-Cafeeiro Leucoptera coffeella (Guérin-Mèneville) (Lepidoptera: Lyonetiidae)? Neotropical Entomology 4: 543-548.

Andrade, F.R. \& Prezoto, F. 2001. Horário de atividade forrageadora e material coletado por Polistes ferreri Saussure,1853 (Hymenoptera, Vespidae), nas diferentes fases do ciclo biológico. Revista Brasileira de Zoociências 3: 117-128.

Bartra, P.C.E. 1974. Biology of Selenaspidus articulatus Morgan and its main biological control. Revista Peruana de Entomologia 17: 60-68.

Barros, E.C.; Bacci, L.; Picano, M.C.; Martins, J.C.; Rosado, J.F. \& Silva, G.A. 2015. Physiological selectivity and activity reduction of insecticides by rainfall to predatory wasps of Tuta absoluta. Journal of Environmental Science and Health 50: 45-54.

Bueno, E.T.; Souza, M.M. \& Clemente, M.A. 2019. The Effect of Forest Fragmentation on Polistinae. Sociobiology 66: 508-514.

Canevazzi, N.C.S. \& Noll, F.B. 2011. Environmental Factors Influencing Foraging Activity in the Social Wasp Polybia paulista (Hymenoptera). A Journal of Entomology 1: 1-8.

Cooper, M.A. 1997. New subgenus Megacanthopus of Mischocyttarus de Saussure (Hym. Vespidae, Polistinae) with a key and three new species. Entomologist's Monthly Magazine 133: 217-233.

Dejean, A.; Corbara, B. \& Carpenter, J.M. 1998. Seleção de locais de nidificação por vespas na Floresta Guaianese. Insectes Sociaux 45: 33-41.

Elisei, T.; Nunes, J.V.; Junior, C.R.; Junior, A.J.F. \& Prezoto, F. 2010. Uso da vespa social Polistes versicolor no controle de desfolhadores de eucalipto. Pesquisa Agropecuária Brasileira 45(5): 958-964.

EMBRAPA, 2019. Estudos socioeconômicos e ambientais: Café arábica corresponde a 70\% e café conilon a 30\% da produção dos Cafés do Brasil em 2019. Disponível em: https://www.embrapa.br/busca-de-noticias/-/noticia/47028493/cafe-arabica-corresponde-a-70-e-cafe-conilon-a-30-da-producao-dos-cafesdo-brasil-em-2019. Acesso em: 21 abr. 2020. 
Fernandes, F.L.; Mantovani, E.C.; Neto, H.B.; Nunes, V.V. 2009. Efeitos de variáveis ambientais, irrigação e vespas predadoras sobre Leucoptera coffeella (Guérin-Méneville) (Lepidoptera: Lyonetiidae) no cafeeiro. Neotropical Entomology 38: 410-417.

Fragoso, D.B.; Jusselino-Filho, P.; Guedes, R.N.C. \& Proque, R. 2001. Proteção de plantas: Seletividade de Inseticidas a Vespas Predadoras de Leucoptera coffeella (Guèr.-Mënev.) (Lepidoptera: Lyonetiidae). Neotropical Entomology 1: 139-144.

Francisco, G.S.; Souza, M.M.; Clemente, M.A. \& Brunismann, A.G. 2018. Substrato Vegetal utilizado para nidificação de vespas sociais (Hymenoptera, Vespidae) em Floresta Decidual. Revista Agrogeoambiental 10(3): 35-45.

Freitas. J.L.; Pires. E.P.; Oliveira, T.T.C.; Santos. N.L. \& Souza, M.M. 2015. Vespas sociais (Hymenoptera: Vespidae) em lavouras de Coffea arabica L. (Rubiaceae) no Sul de Minas Gerais. Revista Agrogeoambiental 7: 67-77.

Gravena, S. 1983. Táticas de manejo integrado do bicho mineiro do cafeeiro Perileucoptera coffeella (Geurin-Meneville,1842): Dinâmica populacional e inimigos naturais. Anais da Sociedade Entomológica do Brasil 12: 61-71.

Hammer, O.; Harper, D.A.T. \& Ryan, P.D. 2005. Past: paleontological statistics software package for education and data analysis. Palaeontologica Electronica 4: 1-9.

Hoshino, A.T.; Bortolloto, O.C.; Hata, F.T.; Ventura, M.U. \& Junior, A.O.M. 2018. Effect of pigeon pea intercropping or shading with leucaena plants on the occurrence of the coffee leaf miner and on its predation by wasps in organic coffee plantings. Ciência Rural 48(3): 1-7.

Jacques, G.C.; Castro, A.A.; Souza, G.K.; Silva-Filho, R.; Souza, M.M. \& Zanuncio, J.C. 2012. Diversity of social wasps in the Campus of the "Universidade Federal de Viçosa" in Viçosa, Minas Gerais State, Brazil. Sociobiology 59(3): 1053-1062.

Jacques, G.C.; Souza, M.M.; Coelho, H.J.; Vicente, L.O. \& Silveira, L.C.P. 2015. Diversity of Social Wasps (Hymenoptera: Vespidae: Polistinae) in an Agricultural Environment in Bambuí, Minas Gerais, Brazil. Sociobiology 62(3): 439-445.

Jonsen, I.D. \& Fahrig, L. 1997. Response of generalist and specialist insect herbivores to landscape spatial structure. Landscape Ecology 12:185-197.

Lawton, J.H 1983. Arquitetura de plantas e diversidade de insetos fitófagos. Annual Review of Entomology 28: 23-39. 
Machado, E.L.M.; Oliveira-Filho, A.; Carvalho, W.A.C.; Souza, J.S.; Tristão, R.A. \& Borém. L.B.A. 2004. Comparative analysis of the structure and flora of the tree-shrub compartment from a remnant forest at Fazenda Beira Lago. Árvore 28:10-12.

Marques, O.M. 1996. Vespas Sociais (Hymenoptera, Vespidae): Características e importância em agroecossistemas. Insecta 3:18-139.

Nais, J. \& Busoli, A. C. 2012. Morphological, behavioral and biological aspects of Azya luteipes Musant fed on Coccus viridis (Green). Scientia Agrícola 69: 81-83. doi.org/10.1590/S0103-90162012000100012.

Naumann, M.G. 1975. Comportamento de enxame: Evidências de comunicação em vespas sociais. Science 189: 642-644.

Nakasuji, F.; Yamanaka, H. \& Kiritani, K. 1976. Predation of larvae of the tobacco cutworm Spodoptera litura (Lepidoptera, Noctuidae) by Polistes wasps. Kontyu 44(20): 5-13.

Oliveira, T.C.T.; Souza, M.M. \& Pires, E.P. 2017. Nesting habits of social wasps (Hymenoptera: Vespidae) in forest fragments associated with anthropic areas in southeastern Brazil. Sociobiology 64(1): 101-104.

Parra, J. R. P.; Gonçalves, W.; Gravena, S.; Marconato, A. R. 1977. Parasitos e predadores do bicho-mineiro do cafeeiro Perileucoptera coffeella (Guérin-Méneville, 1842) em São Paulo. Anais da Sociedade Entomológica do Brasil 6: 138-143.

Pereira, V.S. \& Santos, G.M.M. 2006. Diversity in bee (Hymenoptera, Apoidea) and social wasps (Hymenoptera, Vespidae) community in Campos Rupestres, Bahia, Brazil. Neotropical Entomology 35: 165-174.

Perioto, N.W.; Lara, R.I.R. \& Santos, E.F. 2011. Estudos revelam presença de novos inimigos naturais de pragas da cafeicultura II Vespas predadoras. Pesquisa e Tecnologia 8: 01-06.

Pickett, K.M. \& Wenzel J.W. 2007. Revision and cladistic analysis of the nocturnal social wasp genus, Apoica Lepeletier (Hymenoptera: Vespidae; Polistinae, Epiponini). American Museum Novitates 3562: 130.

Prezoto, F. \& Machado, V.L.L. 1999. Ação de Polistes (Aphanilopterus) simillimus Zikán (Hymenoptera, Vespidae) no controle de Spodoptera frugiperda (Smith) (Lepidoptera, Noctuidae). Revista Brasileira de Zoologia 16: 841-850.

Prezoto, F.; Santos-Prezoto, H.H.; Machado, V.L.L. \& Zanuncio, J.C. 2006. Prey captured and used in Polistes versicolor (Olivier) (Hymenoptera: Vespidae) nourishment. Neotropical Entomology 35(2): 707709. 
Raveret-Richter, M. 1990. Hunting social wasp interactions: Influence of prey size, arrival order, and wasp species. Ecology, Washington 71(3): 1018-1030.

Raveret-Richter, M. 2000. Social Wasp (Hymenoptera, Vespidae) Foraging Behavior. Annual Review of Entomology 45: $121-150$.

Reis, P.R. \& Souza, J.C. 2002. Insetos na folha. Cultivar 4(38): 30-33.

Reis, P.R.; Souza, J.C. \& Venzon, M. 2002. Manejo ecológico das principais pragas do cafeeiro. Informe Agropecuário 23: 83-99.

Richards, O. W. 1978. The social Wasps of the Americas, Excluding the Vespinae. London, British Museum, Natural History. 580p.

Righi, C.A.; Campoe, O.C.; Bernardes, M.S.; Lunz, A.M.P.; Piedade, S.M.S. \& Pereira, C.R. 2013. Influence of rubber trees on leaf-miner damage to coffee plants in an agroforestry system. Agroforestry Systems 87(6): 1351-1362.

Santos, G.M.M.; Silva, S.O.C.; Bichara Filho, C.C. \& Gobbi, N. 1998. Influencia del tamaño del cuerpo en el forrajeo de avispas sociales (Hymenoptera - Polistinae) visitantes de Syagrus coronata (Martius) (Arecaceae). Revista Internacional de Biodiversidad, Oceanologia, y Conservación Universidade de Concepción - Chile 62: 167-170.

Santos, G. M.M.; Santana-Reis, V.P.G.; Resende, J. J.; De Marco, P. \& Bichara Filho, C.C. 2000. Flying capacity of swarm-founding wasp Polybia occidentalis occidentalis Olivier, 1971 (Hymenoptera, Vespidae).

Revista Brasileira de Zoociências 2: 33-39.

Santos, G.M.M.; Filho, C.C.B.; Resende, J.J.; Cruz, J.D. \& Marques, O.M. 2007. Diversity and community structures of social wasps (Hymenoptera: Vespidae) in three ecosystems in Itaparica Island, Bahia State, Brazil. Neotropical Entomology 36: 180-185.

Scalon, J.D.; Avelar, M.B.L.; Alves, G.F. \& Zacarias, M.S. 2011. Spatial and temporal dynamics off coffeeleaf-miner and predatory wasps in organic coffee field in formation. Ciência Rural 41: 646-652.

Souza, M.M. \& Prezoto, F. 2006. Diversity of social wasps (Hymenoptera: Vespidae) in semideciduous forest and cerrado (Savanna) regions in Brazil. Sociobiology 47(1): 135-147.

Souza, M.M.; Louzada, J.; Serrão, J.E. \& Zanuncio, J.C. 2010. Social Wasps (Hymenoptera: Vespidae) as Indicators of Conservation Degree of Riparian Forest in Southeast Brazil. Sociobiology 56(2): 387-396.

Souza, M.M.; Pires, E.P.; Ferreira, M.; Ladeira, T.E.; Pereira, M.; Elpino-Campos, A. \& Zanuncio, J.C. 2012. 
Biodiversidade de vespas sociais (Hymenoptera: Vespidae) do Parque Estadual do Rio Doce, Minas Gerais, Brasil. MG-Biota 5(1): 4-19.

Tomazella, V.B.; Jacques, G.C.; Lira, A.C. \& Silveira, L.C.P. 2018. Visitation of Social Wasps in Arabica Coffee Crop (Coffea arabica L.) Intercropped with Different Tree Species. Sociobiology 65(2): 299-304.

Tuelher, E.S.; Oliveira, E.E.; Guedes, R.N.C. \& Magalhães, L.C. 2003. Ocorrência de bicho-mineiro do cafeeiro (Leucoptera coffeella) influenciada pelo período estacional e pela altitude. Acta Scientiarum: Agronomy 25(1): 119-124.

Vinhote, J.F.C.; Torres, A.F.C.; Dantas, R.T.; Praciano, T.P.; Menezes, R.R.P.P.B.; Souza, D.F.; Brito, T.S.; Lima, F.J.B.; Toyama, M.H.; Magalhães, P.J.; Monteiro, H.S.A. \& Martins-Nunes, A.M.C. 2011. Renal- and calcium-dependent vascular effects of Polybia paulista wasp venom. Journal of Venomous Animals and Toxins including Tropical Diseases 17(2): 199-208.

Zanella, L.; Borém, R.A.T.; Souza, C.G.; Alves, H.M.R. \& Borém, F.M. 2012. Atlantic Forest fragmentation analysis and landscape restoration management scenarios. Natureza \& Conservação 10: 57-63. doi10.4322/natcon. 201201. 